\title{
DEONTOLOGÍA Y CONSECUENCIALISMO: UN ENFOQUE INFORMACIONAL
}

\author{
Rafael Cejudo Córdoba \\ Facultad de Filosofía y Letras \\ Universidad de Córdoba (España) \\ rafael.cejudo@uco.es
}

RESUMEN: Durante los últimos cuarenta años el consecuencialismo ha evolucionado para responder a numerosas críticas, hasta el punto de que hoy comparte rasgos importantes con las teorías deontológicas. El enfoque informacional de Amartya Sen analiza el tipo y la cantidad de información que una estructura ética necesita. Uso este método para determinar cuánto puede aproximarse a la deontología este nuevo consecuencialismo, y cuán difícil puede ser tal expansión. En el contexto del principio del doble efecto, el enfoque informacional permite mostrar que existe una divergencia inevitable entre la deontología y el consecuencialismo. Esta disparidad manifiesta dimensiones genuinas de la razón práctica.

PALABRAS CLAVE: relatividad respecto del agente, razón práctica, Sen, principio del doble efecto, maldad

SUMMARY: For the last forty years, consequentialism has evolved to answer many reviews on it, as far as today it shares important features with deontological theories. Amartya Sen's informational approach analyses the kind and quantity of information needed by an ethical structure. I use this method to ascertain how far this new consequentialism can approach deontology, and how difficult this enlargement could be. In the context of the deontological principle of double effect, an informational approach can show that there is an unavoidable divergence between deontology and consequentialism. That disparity shows genuine dimensions in practical reason.

KEY WORDS: agent relativity, practical reason, Sen, principle of double effect, evil

\section{Deontología y consecuencialismo, estructuras éticas alternativas}

En 1930 escribió Miguel de Unamuno su San Manuel Bueno, mártir, historia de un cura de aldea que, aun habiendo perdido la fe, sigue predicándola a sus parroquianos (Unamuno 1997). Desgarrado por su crisis existencial, el protagonista miente pensando sinceramente que lo hace para que los demás sigan creyendo en el valor de la verdad. Pero Don Manuel es un hombre de principios, y por eso no puede aprobar su mentira aunque gracias a ella otros no mientan. Bajo su ética deontológica o de los principios no es admisible violar una obligación por más que así pudiera evitarse que otros muchos violaran sus correspondientes obligaciones del mismo tipo. En cambio, según una ética sólo atenta a las consecuencias, el comportamiento del santo Don Manuel podría justificarse. Desde luego, tanto 
el consecuencialista como el deontologista prefieren decir la verdad pero, en la circunstancia de que mentir dé lugar a una situación en la que menos personas mientan, el consecuencialista preferirá esto último a lo contrario. El deontologista también considerará correcta una situación en la que más gente sea fiel a sus convicciones, pero a la vez tiene que juzgar correcto hacer algo (no ser hipócrita) que irremisiblemente ocasionará que más gente actúe incorrectamente, que más gente sea hipócrita (Williams 1985; Anderson 1993).

Trataré de demostrar en este artículo que las teorías éticas deontológicas, a diferencia de las consecuencialistas, no pueden perfeccionarse para evitar ese paradójico resultado. A su vez, el consecuencialismo tiene que pagar un elevado precio, quizás demasiado alto, para intentar evitarlo. En la primera sección del artículo se sintetizarán los rasgos que diferencian la deontología del consecuencialismo en cuanto estructuras éticas alternativas. En la segunda sección describiré la forma en que el consecuencialismo se ha "ampliado" asemejándose en algunos rasgos a la deontología, aunque sin renunciar a sus peculiaridades. El núcleo del artículo está en las secciones tercera y cuarta, en las que se exploran las diferencias y las posibilidades de aproximación entre ambas estructuras éticas. Con esa finalidad utilizaré el "enfoque informacional" (informational approach) propuesto por Amartya Sen, aunque los objetivos perseguidos en este artículo me llevan a ampliar el aparato conceptual que él ideó en los años setenta del pasado siglo. El enfoque informacional permitirá pensar hasta qué punto puede ampliarse el consecuencialismo. En el caso de la deontología, el análisis informacional de situaciones donde podría aplicarse el llamado principio del doble efecto, mostrará la diferencia insalvable entre esa estructura ética y el consecuencialismo. El artículo concluye indicando en qué sentido la deontología, el consecuencialismo y la diferencia entre ambos recogen distintas facetas de la razón práctica.

\section{Dos estructuras éticas}

La deontología y el consecuencialismo son dos tipos característicos de teoría ética. En el primer caso, el acento recae en los principios de acción, en las obligaciones que pesan sobre el agente moral (por ejemplo, decir la verdad), mientras que en el segundo lo importante es que algún objetivo valioso se cumpla en el mundo (por ejemplo, que la gente viva más feliz). Estas dos clases de "estructuras éticas", como Mackie (1977) las calificaba, conllevan concepciones diferentes de lo correcto y del valor moral. La expresión "consecuencialismo" traduce 
la voz inglesa "consequentialism". Se debe a Sen (1979b) la idea de distinguir el consecuencialismo como un rasgo del utilitarismo, de modo que éste puede descomponerse en tres elementos: "bienestarismo" (welfarism), o interés en el bienestar individual para definir la utilidad; "ordenación mediante la suma" (sum ranking), consistente en evaluar mediante la suma de la utilidad individual; y finalmente, "consecuencialismo" o tesis de que hay que evaluar las opciones y acciones sólo por los estados de cosas que de ellos se sigan, o dicho a la inversa, que los estados de cosas alternativos se juzgarán meramente en términos de sus componentes, prescindiendo de las intenciones y la identidad de sus responsables. ${ }^{1}$ Así pues, consecuencialismo es una denominación amplia que cubre tanto diferentes modalidades de utilitarismo como otras éticas teleológicas no comprometidas con el principio de utilidad.

El paradigma de ética consecuencialista es el utilitarismo, pero desde los años 1970 asistimos a un constante perfeccionamiento de la teoría utilitarista mediante un debate serio con la ética de los principios (Scheffler 1982; Kagan 1989; Broome 1992; Nino 1992; Baron, Pettit y Slote 1997; Mulgan 2001). Es muy significativo el título que Hare (1993) eligió para un artículo: “¿Podría Kant haber sido utilitarista?" En ese texto consideraba que, con ciertas matizaciones, la respuesta es afirmativa, y hoy Parfit defiende que, si no utilitarista, al menos podría ser consecuencialista (Morgan 2009; Otsuka 2009; Parfit en prensa). En cambio, cuando hacemos la pregunta contraria ("¿podría Bentham haber sido deontologista?"), la respuesta tiene que ser un no rotundo. Y eso depende no sólo del utilitarismo de Bentham, sino de la naturaleza general de la ética deontológica. Las dificultades profundas para acercar entre sí ambas clases de teoría ética son un reflejo del carácter trágico de la vida moral, tal como ilustra la novela de Unamuno.

En el consecuencialismo, lo correcto se define como la maximización de lo bueno, y a su vez esto último es definido independientemente de lo correcto (como utilidad, felicidad, placer o de alguna otra forma). ${ }^{2}$ En esta clase de estructura ética actuar correctamente es hacer algo por nuestro bien o por el bien de los demás (si es que esto último es más importante que mi ventaja particular). Se nos manda, por lo tanto, promocionar lo bueno, conseguir que haya más y no

${ }^{1}$ Mucho antes, Anscombe (1958) había distinguido el consecuencialismo como un rasgo del utilitarismo.

${ }^{2}$ Cfr. Rawls 1971, p. 25. Rawls utiliza la expresión "teleología" en lugar de "consecuencialismo", pero, como acabo de explicar, se está refiriendo precisamente a ese rasgo de las éticas teleológicas que vamos a llamar "consecuencialismo". 
menos de aquello que hace al mundo más valioso. ${ }^{3}$ Por el contrario, la deontología defiende que debemos actuar de cierta manera porque hacerlo así está bien (y no porque hacerlo así cause más bien). Bajo esta otra perspectiva lo correcto se define independientemente de lo bueno: un acto puede ser correcto, y por tanto exigible, aunque no haya buenos motivos para realizarlo (Ross 1930). De la convicción de que existen acciones buenas (o malas) en sí mismas, se sigue el deber de realizarlas (o de evitarlas), un deber cuya corrección no descansa en las consecuencias de la acción, y que por tal motivo Kant (1785) denominaba categórico y Ross (1930) sans phrase.

En el consecuencialismo, el valor de las acciones depende del de las consecuencias producidas o, con más precisión, las acciones son consideradas como desencadenantes de cambios en la realidad, de modo que serán estos cambios lo que realmente habrá que valorar. Desde esta perspectiva, correcto es lo que produce el mayor bien posible, así que no hay nada por sí mismo valioso en el acto correcto, y la noción de "corrección" (entiéndase moral), que es lo distintivo de la deontología, queda aquí disuelta en la de maximización o promoción de lo bueno. A su vez, para explicar el valor "bueno" que el consecuencialista atribuye a ciertos estados de cosas, no podemos recurrir a la idea de corrección, pues caeríamos en una falacia circular: si las acciones son correctas por sus consecuencias, éstas no pueden valer por ser correctas. La solución está en defender una teoría pluralista del valor, de modo que el valor moral de promocionar al máximo lo bueno dependa del valor no moral de eso bueno. Dicho de otra manera, el placer, la felicidad o el bienestar son buenos aparte del mandato moral que nos exige aumentar su presencia real. ${ }^{4}$ Por el contrario, las teorías con estructura deontológica se basan en que las acciones tienen cierto valor específicamente moral, un valor peculiar o deóntico (Scanlon 2001; Zimmerman 2007), que no está causado por sus consecuencias ni puede explicarse totalmente mediante ellas.

Puesto que la deontología atribuye un valor originario a las acciones y el consecuencialismo lo atribuye a las configuraciones objetivas

${ }^{3}$ Dado que la moralidad consiste en fomentar lo bueno en el mundo, la corrección será la evaluación de nuestro éxito en dicha tarea y, por consiguiente, en el balance entre el bien que hemos producido y el mal que no hemos logrado evitar. Decía Frankena (1973, p. 14) al respecto que en la teleología "un acto es correcto si y sólo si tal acto o la regla que le corresponde produce, producirá probablemente, o se ha planeado que produzca un balance de lo bueno sobre lo malo al menos tan positivo como el de cualquiera de las alternativas disponibles; un acto es incorrecto si y sólo si no ocurre lo anterior" (las cursivas son del autor; la traducción es mía).

${ }^{4}$ Ésta es la solución de Frankena (1973), seguida por otros autores como Darwall (2003) en su compilación de ensayos sobre el consecuencialismo. 
del mundo, a los estados de cosas usando la expresión wittgensteiniana, otra forma de captar sus diferencias es mediante el papel que desempeña el sujeto en cada modelo. Esta faceta es especialmente importante puesto que, al fin y al cabo, la deontología y el consecuencialismo son perspectivas alternativas desde las cuales encarar los problemas morales. Tengamos en cuenta que en tales asuntos no siempre actuamos como jueces u observadores externos, sino que en muchas ocasiones, las más importantes, nos toca ser agentes y decidir cuál es la solución mejor o la menos mala. En general, las estructuras éticas consecuencialistas, dejando aparte lo que enseguida añadiremos, son neutrales respecto del agente, pues el juicio moral de una elección posible será el mismo ya seamos los agentes que realizan la elección, ya seamos meros observadores externos. ${ }^{5}$ Puesto que la moralidad de la acción reside en el grado en que fomenta la bondad del mundo, es indiferente quién la realiza. La explicación completa de esta característica de las éticas consecuencialistas es compleja y excede los objetivos de este artículo. Apuntemos sólo su relación con la naturaleza no moral de aquello que se valora en las consecuencias (el placer o el bienestar son, como tales, buenos) (Darwall 2006, pp. 1-7), y sobre todo con el vínculo que el consecuencialismo establece implícitamente entre racionalidad y universalidad (si algo es universalmente bueno, cualquier sujeto racional lo preferiría) (Salcedo 1991).

La relatividad respecto del agente es distintiva de la deontología, y consiste en admitir que el juicio moral de un mismo hecho puede ser distinto si lo realiza el agente y si lo realiza un observador externo. $\mathrm{Y}$ es que la deontología niega que la corrección moral dependa de un valor no moral apreciable al margen de nuestra situación particular. Al juzgar una alternativa, lo relevante para el consecuencialismo es, por ejemplo, el dolor que se produce en un caso $\mathrm{u}$ otro. Por el contrario, lo importante en la deontología es el daño producido por el sujeto que actúa. La corrección moral reside en la acción realizada y la descripción de ésta tiene que incorporar al agente responsable de la misma. La relatividad respecto del agente implica considerar la posición que éste ocupa en el estado de cosas, lo cual incluye su posición en las cadenas causales determinantes del mismo. La obligación deontológica es que yo no mate, y no simplemente evitar un fallecido

\footnotetext{
${ }^{5}$ Siguiendo la distinción que Nagel (1980) había propuesto entre razones objetivas y subjetivas, Parfit (1984, p. 143) propuso esta distinción entre razones neutrales respecto del agente (agent-neutral) y relativas respecto del agente (agent-relative), definiéndolas como aquellas que son "razones para todo el mundo", frente a las que son razones "sólo para el agente".
} 
más en el mundo. ${ }^{6}$ Como enseguida explicaremos, el consecuencialismo puede tener en cuenta, al juzgar el estado de cosas, el mérito o la culpa del agente que lo ha causado, pero esa consideración se incluye en otra más general que abarca los demás aspectos del estado de cosas. Por eso, una acción execrable (desde cualquiera de los dos modelos) puede formar parte de una situación que el consecuencialismo a pesar de todo aprobaría. Al respecto dice Sen (1983, p. 130) que este último se caracteriza por "la insobornable necesidad de ir hasta cualquier parte a la que la evaluación del estado de cosas nos lleve". 7

\section{Consecuencialismo ampliado}

Hasta aquí he presentado la deontología y el consecuencialismo como tipos contrarios y alternativos de teoría ética, y defiendo de hecho que existe una diferencia profunda e insalvable entre ambos. Sin embargo, no deberíamos pensar que el consecuencialismo se desentiende de las acciones ni que la deontología se olvida de las consecuencias. Sólo las interpretaciones triviales de las teorías éticas más representativas de cada tipo (el utilitarismo y el kantismo) abonan esa percepción equivocada. No debemos leer la máxima fiat justitia et pereat mundus en el sentido de que las consecuencias, el "mundo", sea algo de lo que el buen kantiano pueda prescindir. Más bien sucede que el kantiano desea (esto es, la deontología exige) que el mundo sea justo, que ciertas consecuencias sucedan en el mundo, y que éste sea de cierta manera debido a ello. La deontología defiende que las acciones tienen un valor propio, y esto no impide aceptar que la ontología de las acciones incluye tanto el agente que las realiza, como los propósitos que persigue. Se trata, por lo tanto, de que el mundo sea de cierta manera debido a que nosotros hemos actuado en él

${ }^{6}$ La introducción del punto de vista del agente supone (Parfit 1979, p. 28), en suma, cambiar la descripción de lo que se hace (que los hijos sean cuidados por sus padres — neutral respecto del agente-, o que yo cuide de mis hijos — relativo-), y por consiguiente, también de las consecuencias (que los hijos no mueran de hambre -neutral一, frente a que mis hijos no mueran - relativo-).

${ }^{7}$ Al igual que Larmore (1987, p. 146) al comentar la relatividad del agente como distintiva de la deontología, Sen utiliza el ejemplo del dilema de Jim, ideado por Williams (1973, p. 117): si un terrorista nos propone el macabro dilema de elegir que él mate a diez personas o que nosotros asesinemos a una para que las otras nueve vivan, el consecuencialismo tendrá que elegir la segunda opción porque, aun admitiendo que asesinar es malo, la corrección de nuestra acción se refiere al estado de cosas resultante. 
apropiadamente. ${ }^{8}$ Adecuadamente entendida, la deontología es una ética de la responsabilidad (Weber 1992).

De manera similar, el consecuencialismo no siempre se despreocupa de las acciones que causan los estados de cosas. Por eso, las definiciones fáciles aquí también son inútiles. Tomemos por ejemplo la que en cierta ocasión propuso Pettit (1993, p. xiii): “de manera simple, el consecuencialismo es la teoría de que la manera de especificar si una particular elección es la correcta para el agente que la realiza consiste en considerar las consecuencias relevantes de la decisión, en advertir los efectos de tal decisión en el mundo". Pero la deontología también suscribe lo mismo, de modo que debemos abandonar las definiciones superficiales. No obstante, existen realmente diferencias insalvables entre ambos modelos de teoría ética. A diferencia de la deontología, y ésta es una de las cuestiones que querría probar, el consecuencialismo puede ampliarse o enriquecerse para incorporar ciertos rasgos atractivos de la deontología sin abandonar la concepción general que líneas atrás ya ha sido expuesta. De hecho, así ha sucedido en el llamado "consecuencialismo amplio" o "neoconsecuencialismo". 9

El consecuencialismo amplio ha surgido de una crítica al utilitarismo, de modo que consiste en un consecuencialismo ampliado con relación a este último. ${ }^{10} \mathrm{El}$ utilitarismo defiende que lo bueno consiste en bienestar, cualidad que se predica de los estados de cosas. Las acciones correctas serían las que promueven el mayor bienestar, y no tienen más valor que el de su contribución a este objetivo. Asimismo es irrelevante que el agente sea uno u otro, pues lo importante es el resultado de su acción. Por ejemplo, según el utilitarismo, es indiferente que sea su padre o la socorrista quien salve al niño que se ahoga, y es mejor que el padre salve a dos niños desconocidos, aunque su hijo se ahogue, antes que salvar sólo a su hijo. Esta clase de razonamiento moral es tosco a ojos del neoconsecuencialista, quien defiende que la deliberación tiene que seguir derroteros más complejos. Para

${ }^{8}$ A este respecto, dice Williams (1985, p. 56): "lo que deseamos es que el resultado se haya producido porque lo queríamos, porque creíamos ciertas cosas, y porque actuamos como lo hicimos sobre la base de esos deseos y creencias" (la traducción es mía).

${ }^{9}$ La primera denominación se debe a Sen (1983), y Salcedo (1991) sugiere la segunda. Sobre las principales propuestas neoconsecuencialistas, cfr. Sen 1982, 1983, 1993, 2000, Railton 1984, Conly 1985, Scheffler 1988, Broome 1991, Jackson 1991, Griffin 1992, Kagan 1998, Darwall 2003, Carrasco 2002.

${ }^{10}$ En realidad, "consecuencialismo" a secas es suficientemente preciso en muchos contextos, puesto que si usamos "consecuencialismo" en lugar de "utilitarismo" es porque consideramos que ambos términos no son sinónimos. En este sentido, Walsh (1996, p. 70) habla de “consecuencialismo "puro" " (la traducción es mía). 
empezar, es posible incluir la acción realizada en la descripción del estado de cosas consecuente, y tener en cuenta el valor de esa acción en el balance general de las consecuencias. Esto es, el juicio incluirá el bienestar producido en unos, el malestar ocasionado en otros, las reglas violadas — si defendiéramos un utilitarismo de las reglas-, la utilidad total o media... así como el valor de la acción causante de todo ello (Prichard 1912; Scheffler 1982; Broome 1991). En segundo lugar, también puede tenerse en cuenta la identidad del agente, cosa lógica si vamos a considerar la propia acción (Scheffler 1988; Darwall 2003; Lara y Francés 2004). Si la consecuencia va a ser que yo me salve de un ataque, no es irrelevante que mi atacante muera a manos de la policía o que sea yo mismo quien lo mate; si la consecuencia va a ser que mi hija se salve de morir ahogada, no es irrelevante que precisamente sea yo quien la salve.

Adviértase que el neoconsecuencialismo no renuncia a que lo correcto se defina según los estados de cosas, pues el valor de la acción y la identidad de su agente no son el criterio para decidir sobre el mismo, sino sólo parte de su descripción. El consecuencialismo amplio tendrá esos factores en cuenta como parte del valor de las consecuencias, pero nada más, puesto que en él "la corrección de las acciones no tiene más sentido real que el que se refleja en los estados de cosas", como explica Sen (1983, p. 130). Esta solución se basa en la distinción consecuencialista entre lo correcto y lo bueno: las acciones correctas siguen siendo las que provoquen el mayor bien; no obstante, lo bueno incluye ahora, citando de nuevo a Sen (1983, p. 130), "el valor o disvalor que las acciones tienen por sí mismas".

Se trata de una nueva aplicación de lo que McNaughton y Rawling (1991) llamaron el "aspirador consecuencialista": la posibilidad que tiene el consecuencialismo de "ampliarse" todo lo que sea necesario incorporando en su concepción de lo bueno todos aquellos aspectos que le sean criticados. Continuando con el símil, en este caso el aspirador no se aplica al concepto de bueno, sino a la descripción del estado de cosas: se trata de "succionar" el valor intrínseco de las acciones, característico de las posiciones deontológicas. La ampliación del consecuencialismo se traduce en la posibilidad de contar con descripciones cada vez más finas o refinadas de los estados de cosas o, lo mismo, de las consecuencias que sean el caso. De esa manera, por ejemplo, es posible dar cuenta con más justicia de los actos supererogatorios, cuyo mérito debe ser especial. Pensemos en el caso de quien decide salvar de morir ahogado a un desconocido arriesgando su propia vida. En el supuesto de que la probabilidad 
de morir fuera la misma y uno de los dos muriera, el estrecho consecuencialismo benthamita evaluaría por igual que fuera el héroe o la víctima. En cambio, el neoconsecuencialismo puede dar cuenta del valor intrínseco de un sacrificio desinteresado como parte de la valoración global del estado de cosas resultante. Análogamente, la descripción de las consecuencias puede distinguir entre, por ejemplo, que los hijos no mueran ahogados, que mis hijos no mueran ahogados y, por último, que sea yo quien salve a mis hijos de ello, asignando valores distintos a cada uno de tales estados de cosas. O también, las consecuencias pueden describirse recogiendo si se ha violado o no un derecho, y además si el cumplimiento del mismo conlleva que su titular alcance sus objetivos o si, por el contrario, el respeto del derecho es meramente formal.

Gracias a ello, el neoconsecuencialismo representa con mayor fidelidad las sombras de la vida moral. En contra de la tesis utilitarista, no siempre lo correcto coincide con lo óptimo, y esto es algo que el consecuencialismo amplio sí puede captar, pues cuando reconocemos que las acciones tienen un valor moral intrínseco que contribuye a la bondad de las consecuencias, llamarlas "óptimas" a pesar de que sea mala la acción que las causa es meramente una cuestión de palabras. Además, el consecuencialismo amplio permite matizar el reduccionismo utilitarista de la razón instrumental. Caemos en el mismo considerando que la racionalidad surge del hecho empírico de que la conducta humana está orientada hacia metas, y de que, por consiguiente, la acción racional es básicamente teleológica (Harsanyi 1982). Según esta concepción, la "acción respecto de valores" típica de la deontología (Weber 2002) queda condenada a las fronteras de la racionalidad, puesto que en ella nada se maximiza. Por el contrario, si reconocemos que las acciones pueden tener valor independientemente de los resultados a los que den lugar, avanzamos hacia la recomposición de la razón práctica.

\section{Análisis informacional del consecuencialismo ampliado}

Todas esas ventajas tienen un alto costo en términos de la complejidad que el consecuencialismo ampliado debe soportar, puesto que exige una considerable ampliación de la base informacional de la teoría ética consecuencialista. La base y las constricciones informacionales son distinciones propuestas por Sen que pueden ser de gran utilidad para establecer los límites del neoconsecuencialismo. ${ }^{11}$ En cada teoría

${ }^{11}$ El enfoque informacional fue concebido por Sen $(1974,1977)$ como un instrumento para analizar y criticar la metodología de la economía del bienestar, pero 
ética han de existir reglas estructurales, normalmente implícitas, que definan la clase de información de la que depende directamente un juicio moral que sea conforme con la teoría. A ese tipo de información Sen lo denomina base informacional (informational basis). A la vez, tales reglas estructurales establecen qué clases de información no van a tener efecto sobre los juicios morales realizados. Tales constricciones informacionales (informational constraints) tienen la virtud de categorizar la información potencialmente relevante, y por este motivo indican cuáles son los compromisos axiológicos de una teoría, o qué tipo de cosas tienen valor bajo su criterio. Por ejemplo, en el utilitarismo benthamita (Bentham 2005, pp. 11 y ss.) la base informacional está integrada por información sobre el placer individual, sobre el agregado total de placer individual, y sobre la relación entre estados de cosas y variaciones intraindividuales de placer. En este caso, las constricciones informacionales son muy amplias: los grados y las diferencias de placer no cuantificables, la distribución del total de placer individual, la intención de los agentes causantes de alteraciones en los estados de cosas, etcétera. En el caso del utilitarismo de los actos, una constricción informacional es la información sobre las normas que pudieran resultar aplicables, puesto que, según esta teoría, la corrección de un estado social depende de la utilidad de los actos realizados, estén o no de acuerdo con las normas vigentes. En cambio, esa constricción informacional no rige en el utilitarismo de las reglas (Brandt 1959). En la deontología rawlsiana — la justicia como equidad - , una constricción informacional es, al contrario que en el utilitarismo, el total de utilidad del estado social, y otra es el total de excelencia moral conseguido, pues según Rawls (1971, p. 94), una vez que los principios de justicia han diseñado la estructura básica de la sociedad, "no se hacen preguntas ni sobre los totales de satisfacción, ni sobre los de perfección".

El tipo y la cantidad de información que una teoría requiere permiten determinar su alcance y su ámbito de aplicación, mientras que la clase de información que excluye manifiesta cuáles son sus compromisos axiológicos. La amplitud de la base informacional varía en cada caso concreto (las mayores constricciones informacionales están en el utilitarismo benthamita, son menores en el milliano, y son mucho menores en el consecuencialismo de Sen, por ejemplo). En realidad, el neoconsecuencialismo es resultado de una ampliación de la base informacional del utilitarismo clásico, y por consiguien-

pronto lo aplicó también a la filosofía moral, concretamente a la crítica del utilitarismo y de la "justicia como equidad" rawlsiana (Sen 1979a). 
te, de reducir sus constricciones informacionales. Más aún, lo que voy a llamar profundidad del análisis informacional queda abierto en el neoconsecuencialismo, puesto que se requieren descripciones sucesivamente más precisas de las consecuencias para poder incluir todos los matices de la relación entre agentes y acciones. Por eso, la amplitud posible de la base informacional en las estructuras neoconsecuencialistas está indeterminada. Ahora bien, con ello la complejidad de la teoría va en aumento, y es natural suponer que dicha tendencia debe tener un límite.

Por un lado, la disminución de las constricciones informacionales tiene un final en los compromisos o intuiciones básicos del consecuencialismo, más allá de los cuales la teoría dejaría de entenderse como consecuencialista en un sentido propio (sobre este asunto volveré enseguida). Por otro lado, la ampliación de la base informacional tiene que pagar el precio de la complicación teórica. Ambos criterios marcan una frontera de ampliación de la base informacional que refleja la potencia de la teoría, esto es, el éxito de su estrategia para encarar los problemas morales. Téngase en cuenta que las constricciones informacionales dirigen nuestra atención hacia los aspectos más prácticos de la aplicabilidad de los principios, puesto que las dificultades para conseguir toda la información requerida pueden hacer imposible el empleo de un principio por lo demás correcto. En este sentido, señala Sen (1979a, p. 126) que "la utilidad de los principios tiene que juzgarse en términos del contexto informacional, y en este aspecto lo mejor puede ser en realidad enemigo de lo aceptable".

Unas constricciones informacionales excesivas impedirán que los juicios morales proporcionados por la teoría discriminen entre diferencias relevantes, y constricciones demasiado laxas harán que la complejidad de la teoría sea inmanejable. Cada teoría puede ofrecer uno o varios principios con los que formular juicios sobre acciones o estados sociales (por ejemplo, en el utilitarismo sólo está el principio de utilidad, mientras que en la justicia como equidad están los dos principios de justicia). A su vez, cada principio permite establecer una jerarquía, más o menos completa, de las acciones o consecuencias bajo su consideración. Si $X$ son todos esos casos y se dispone de toda la información necesaria, la jerarquización de los mismos será todo lo completa que el principio permite. Ahora bien, para conseguir esta jerarquización plena es conveniente aplicar un criterio de economía informacional que limite la complejidad. Con esta finalidad, Sen (1979a, pp. 119-120) considera que la información disponible es adecuada para un principio dado $p$ cuando basta para que el 
principio proporcione una jerarquización plena, y es eficiente cuando además es la mínima indispensable.

Pero a estas cualidades metaéticas deberíamos añadir la manejabilidad de la teoría, entendida como la posibilidad de adquirir la información necesaria, y de razonar sobre ella con una capacidad intelectual normal. Si la información eficiente no es manejable, la teoría se convertirá en una mathesis universalis, en un juguete leibniziano cuya utilidad se difiere a un impreciso futuro en que humanos (o máquinas) seamos más plenamente racionales, pues aunque consideráramos que las teorías neoconsecuencialistas se refieren a la fundamentación abstracta en lugar de a la deliberación concreta, una teoría ética cuya información eficiente sea más manejable que la de otra resulta prima facie preferible. Y ésta es una cuestión independiente de que la teoría mejor tenga una estructura consecuencialista, deontológica o mixta.

\section{Análisis informacional de la deontología}

La ambición del neoconsecuencialismo es dar cuenta de la deliberación racional en aquellos casos en los que el modelo deontológico parece ser más apropiado (derechos y obligaciones, vínculos personales, prohibición del "maquiavelismo" y de los actos terribles). Aunque en comparación con el consecuencialismo utilitarista su éxito en esos casos es indudable, la diferencia con la deontología subsiste de todas formas. Trataré de demostrar que así debe ser por más que se amplíe el consecuencialismo. A diferencia de este último, la concepción deontológica cierra o fija la profundidad del análisis informacional. De un mismo estado de cosas caben diferentes descripciones, y esto permite la riqueza de matices característica del consecuencialismo ampliado. ${ }^{12}$ Sin embargo, en el caso de la deontología hay un nivel de detalle del estado de cosas más allá del cual descripciones informacionalmente más ricas no alteran la evaluación deontológica de la acción, por lo que son redundantes desde el punto de vista ético. Ese nivel de precisión descriptiva, y por tanto de profundidad en el análisis informacional, es justo el requerido para la caracterización adecuada de la acción objeto de la evaluación deontológica, de modo que debemos detallar la situación hasta el punto en que la acción en cuestión merezca ya un juicio definitivo sobre la responsabilidad que el agente tiene en la misma.

\footnotetext{
${ }^{12}$ Foot (1985) ha argumentado que, puesto que de un estado de cosas caben distintas descripciones, su valoración moral es relativa, lo que pone en duda todo el consecuencialismo. Pero no es esta línea la que desarrollaré aquí.
} 
Consideremos el siguiente caso (Quinn 1989): una mujer sufre un cáncer de útero por el que su vida corre peligro de muerte si no se le extirpa dicho órgano. Añadamos que dicha mujer está en un avanzado estado de gestación, aunque no lo suficiente como para que su hijo pueda sobrevivir por sí solo, ni tampoco para que pueda esperarse a que ella dé a luz. De hecho, esperar hasta el término del embarazo sería fatal para la madre y el hijo. Los médicos operan a la paciente. ¿Qué han hecho los médicos exactamente: han extirpado un útero enfermo, han salvado a la madre, han provocado el aborto del feto? ¿Todas esas cosas a la vez o sólo algunas de ellas? El interés que los casos de este tipo tienen para el razonamiento moral reside en que en ellos existen consecuencias conocidas e indeseables para el agente (la muerte del feto), que a la vez son resultado inevitable de sus acciones (extirpar el útero de la madre).

En casos como éste, las éticas deontológicas pueden invocar el llamado principio del doble efecto, formulado originalmente por Tomás de Aquino (1954, p. 536): "nada impide que de un solo acto haya dos efectos, de los cuales uno solo es intencionado y el otro no. Pero los actos morales reciben su especie de lo que está en la intención y no, por el contrario, de lo que es ajeno a ella." En consecuencia, los médicos son responsables de la salvación de la madre, pero no de la muerte del feto, efecto éste no querido por más que ellos lo supieran inevitable. La tendencia general del consecuencialismo es rechazar la validez del principio del doble efecto (Hart 1968; Mackie 1977). En el caso indicado, el motivo es que, al margen de las acciones e intenciones de los médicos, lo que se constata es que las consecuencias de la intervención son la extirpación de un cáncer y la muerte de un feto, y a la mayor o menor bondad de tal estado de cosas es a lo que hay que atenerse. ${ }^{13}$

El principio del doble efecto es una regla exculpatoria cuya invocación es problemática para muchos autores (Griffin 1996; Allison 2001; Scanlon 2008), pero justamente ese carácter polémico permite ilustrar mi tesis sobre la profundidad del análisis informacional en la

${ }^{13}$ Anscombe (1958, p. 12) considera precisamente que el rechazo del principio es el acta de nacimiento del consecuencialismo: "El rechazo de cualquier distinción, en lo referente a la responsabilidad, entre las consecuencias previstas y las perseguidas, no se debió a que Sidgwick desarrollara ningún 'sistema de ética'. Sidgwick hizo esta importante distinción por sí misma y en interés de cualquiera. Considero plausible sugerir que esta distinción de Sidgwick explica la diferencia entre el desfasado utilitarismo y lo que yo llamo consecuencialismo, el cual lo distingue a él y a cualquier filósofo moral académico inglés posterior a él" (las cursivas son de la autora; la traducción es mía). 
deontología. Consideremos un caso parecido al anterior en el cual los médicos, para salvar la vida de la madre, inevitablemente tienen que practicar una craneotomía en el feto, lo cual ocasionará su muerte irremisiblemente (Quinn 1989). ¿Se trata de una situación como la precedente? Esto es, ¿debemos describirla de la misma manera, o en este caso los médicos han hecho algo distinto? Para declarar la acción de los médicos incorrecta en alguna medida, o para sostener que ellos han matado al feto, desde la deontología se requiere una descripción que incorpore la(s) acción(ones) en cuestión como intencionales, y donde el propósito de los agentes al realizarlas tenga que ver con el bien y el mal. Si no es posible una descripción así, entonces la parte del estado de cosas objeto de polémica (la muerte del feto) será un efecto "previsto pero no querido" sobre el que no hay responsabilidad. La clave está en si la descripción correcta es precisamente aquella que manifiesta la responsabilidad que el sujeto reconoce y se atribuye. $^{14}$

Según Anscombe (1957), la descripción de una acción es, en primer lugar, un conjunto de enunciados verdaderos. En los ejemplos que utilizamos, es verdadero que los médicos han extirpado el útero o que han roto el cráneo del feto, pero no lo sería decir, en el segundo caso, que un elemento punzante ha penetrado en la cabeza del feto alterando la estructura craneal. Simplemente eso no es lo que ha sucedido. En segundo lugar, en el caso de una acción intencional existe una respuesta a la pregunta "¿por qué estás haciendo eso?" que no es posible eludir pretendiendo no haber tenido intención de hacer algo que era un medio para los propios fines confesados. Por ejemplo, no puede rechazarse la pregunta "¿por qué estáis destruyendo el cráneo del feto?", respondiendo "no hacemos tal cosa; simplemente estamos salvando a la madre", o respondiendo "simplemente estamos penetrando el cráneo con un elemento punzante", cuando su propio diagnóstico ha reconocido que la rotura del cráneo del feto es necesaria para la supervivencia de la madre.

Y, sobre todo, en el caso de la extirpación del útero no es correcta una descripción de lo que los médicos han hecho que incluya una acción cuyo propósito sea matar al feto, cosa que sí puede hacerse en este segundo caso. En el primer tipo de operación los médicos realizan una acción (una histerectomía) que entraña inexorablemente la

${ }^{14}$ Hay una conexión intrínseca entre el valor deóntico de la acción y el agente que la produce, cosa que subyace en la relatividad del agente (frente a la neutralidad consecuencialista). Por eso Kant (1993, p. 464 A 534/B 562) consideró que la libertad práctica que permite el mérito y la culpa se fundamenta en la libertad trascendental por la que podemos concebir al sujeto como agente y no sólo como efecto. 
muerte del feto, pero sobre cuyo porqué no sería verdadero contestar "hemos hecho una histerectomía para matar al feto". En cambio, en el segundo caso se realiza una acción distinta (una craneotomía) que entraña inexorablemente la muerte del feto, pero sobre cuyo porqué los médicos sí deben contestar "hemos hecho una craneotomía para matar al feto". Es redundante que el estado de cosas del que ambas acciones forman parte se describa con más o menos precisión (podrían ofrecerse páginas y páginas sobre la cirugía de ambas intervenciones), una vez que la información incluida baste para justificar las respuestas indicadas.

En el planteamiento deontológico, lo determinante no es el estado de cosas del que la acción forma parte, sino la propia acción, o con más precisión, su valor deóntico, y éste cierra el nivel de profundidad del análisis informacional requerido. Llegados a ese punto en el que la intención de los agentes ha sido suficientemente clarificada, la evaluación del estado de cosas se convierte en básica, puesto que mayor información no hará que el juicio moral cambie. ${ }^{15}$ Imaginemos el caso de matar en defensa propia, a partir del cual Aquino propuso el principio del doble efecto: si determinamos que la intención del sujeto era matar a su atacante, al margen de defenderse, la evaluación del estado de cosas que abarca tanto su acción como la muerte del oponente habrá de ser negativa, con independencia de que se descubriera que la verdadera causa de la muerte no había sido la repulsión del ataque, sino una enfermedad contraída mucho antes, y que justo entonces llegó casualmente a un desenlace fatal.

\section{Conclusiones}

La aplicación del enfoque informacional a la contraposición entre deontología y consecuencialismo proporciona conclusiones sobre dos asuntos: por una parte, sobre las posibilidades de superar esa vieja escisión de la teoría ética, y por otra, sobre las diferencias en cuanto al bien y el mal moral propias de consecuencialismo y deontología. Comenzando por lo primero, hemos explicado el neoconsecuencialismo en términos de una ampliación de la base informacional del

${ }^{15}$ Sen (1967, p. 50) denominó básicos a aquellos juicios valorativos inalterables bajo cualquier revisión de los supuestos fácticos del caso, y ponía el ejemplo de condenar el asesinato. De todas formas aquí no utilizo básico exactamente en el mismo sentido, porque la deontología no defiende que los juicios morales sean independientes de toda clase de información, ni por tanto de todo lo referente a las consecuencias (cosa que sí defendía Sen en ese artículo de finales de los sesenta). Sucede, más bien, que en la deontología la información fáctica es necesaria y relevante hasta cierto punto más allá del cual el juicio en cuestión se hace básico. 
utilitarismo, de modo que los juicios sobre los estados de cosas ahora incluyen información específica sobre las acciones. Concretamente, deja de ser una constricción informacional la relación entre las acciones causantes de los estados de cosas y sus agentes. Además, el neoconsecuencialismo rechaza que todo el valor de una acción esté vertido en sus consecuencias.

El creciente desarrollo de este planteamiento prueba que en realidad la estructura consecuencialista no estaba limitada según las fuertes constricciones informacionales del utilitarismo. Por el contrario, la que he llamado profundidad del análisis informacional está abierta: en principio es posible ampliar la base informacional indefinidamente para perfeccionar la ética consecuencialista. Pero, aunque conceptualmente la profundidad del análisis informacional pueda ser indefinida, formalmente existen restricciones. A este respecto he introducido los conceptos de frontera de ampliación de la base informacional de una teoría ética, y de manejabilidad de la misma. Quien esté familiarizado con las propuestas neoconsecuencialistas convendrá en la extrema complicación introducida en la descripción, y por lo tanto en la evaluación, de las consecuencias. ${ }^{16}$ A igualdad de condiciones, la manejabilidad de la teoría como instrumento explicativo de la deliberación moral marca una frontera de ampliación. Por otro lado, si relajamos excesivamente las constricciones informacionales del consecuencialismo, también cruzaremos esa frontera, pues habremos perdido sus rasgos distintivos. En el caso de las estructuras deontológicas, la situación es diferente, puesto que la frontera de ampliación no solamente tiene que ver con cuestiones formales, sino también con conceptuales. La posibilidad que tienen las teorías deontológicas de invocar el principio del doble efecto prueba que el nivel del análisis informacional aquí está fijado por la concepción misma de la deontología, ya que el nivel de información es justamente el requerido para recoger el vínculo entre la acción intencional y sus consecuencias.

Esta diferencia conceptual respecto del posible nivel de análisis informacional en uno y otro tipo de teoría ética se debe a una distinta visión de cuál es el núcleo de la moralidad y de la racionalidad práctica. Tanto en la deontología como en el consecuencialismo, la bondad o maldad moral de una acción es su (grado de) corrección. En las éticas consecuencialistas, como al principio se dijo, este valor moral depende de otros no morales (placer, bienestar, utilidad), pues

${ }^{16}$ Es el caso de Sen 1993 y 1982. 
consiste en la promoción de estos últimos. ${ }^{17}$ En el consecuencialismo estrecho, la bondad (y también la maldad) de una acción es una calificación relativa, porque depende de la consideración global del estado de cosas de cuya descripción forma parte. En cambio, como la teoría neoconsecuencialista reconoce el valor deóntico de la acción, la especificidad moral de esta última no queda absorbida por el valor no moral del conjunto de las consecuencias. Sin embargo, y esto es lo que ahora quiero destacar, lo importante en el consecuencialismo ampliado no es la bondad ni la maldad de las acciones, sino su preferibilidad a partir del cómputo global del estado de cosas en el que se inscriben. Puesto que todavía en el neoconsecuencialismo una acción perversa puede ser racionalmente preferible, tenemos que concluir que esto último, la preferibilidad, y no la bondad/maldad moral, es lo peculiar de la concepción de la racionalidad práctica implícita en todas las éticas consecuencialistas. ${ }^{18}$

Una acción incorrecta, perversa, puede ser la racionalmente preferida de entre las alternativas factibles si da lugar a las mejores consecuencias. La preferibilidad de la acción será mayor o menor dependiendo del juicio más general que nos merezca el estado de cosas. Tal como hemos visto más arriba, ese juicio depende de la profundidad del análisis informacional mediante el cual obtenemos descripciones más o menos refinadas del estado de cosas evaluado. Una alternativa inicialmente rechazable puede cambiar de signo gracias a una ampliación de la base informacional, gracias a un mejor conocimiento de los hechos del mundo con los que esa acción mantiene vínculos consecuenciales. Con ello, la racionalidad práctica neoconsecuencialista acorta la distancia entre hechos y valores, puesto que la acción moralmente preferible también depende de información no moral. ${ }^{19}$ Pero este avance en la recomposición de la unidad de la razón tiene

\footnotetext{
${ }^{17}$ Decimos "promoción”, porque, en el consecuencialismo ampliado no siempre se trata de maximizar el total de la utilidad o el valor que sea el caso. También podría minimizarse la desutilidad, maximizarse la utilidad media, o considerar de algún otro modo la equidad de la distribución.

${ }^{18}$ En su crítica del consecuencialismo, Foot (1985, p. 198) indica que el atractivo de éste reside en que no puede ser racional preferir lo peor: "Si hay un estado de cosas perjudicial en el que se ejecuta una de esas acciones, habrá presumiblemente un estado de cosas peor en el que varias de ellas sean realizadas. ¿Y no ha de ser irracional preferir el estado de cosas peor en lugar de el mejor?" (la traducción es mía). Como enseguida precisaré, la respuesta de la deontología es que, en algunos casos así, lo racional es no preferir, o que preferir no es racional.

${ }^{19}$ Según Putnam (2002), esto es lo que Sen trataba de conseguir con la diferencia entre juicios básicos y no básicos frente a la oposición entre compulsivos y no compulsivos.
} 
sus inconvenientes. Tal como he dicho, en el neoconsecuencialismo la preferibilidad de una acción es cosa distinta de su corrección. Una acción incorrecta, perversa, puede ser racionalmente preferible cuando contribuye suficientemente a la bondad de las consecuencias. En cambio, en la ética deontológica subyace la noción de que preferir el mal siempre es irracional, aunque tal elección sea muchas veces inevitable. Es ahí donde reside la tragedia de la vida moral, y no en la disparidad entre principios y consecuencias. El hecho de que la profundidad del análisis informacional tenga que cerrarse en la deontología, de modo que más allá de cierto nivel de detalle la información fáctica adicional sea irrelevante, tiene consecuencias metodológicas en el sentido de marcar una diferencia sustancial con respecto al proyecto neoconsecuencialista. Pero, sobre todo, significa la heterogeneidad entre el valor deóntico y el mundo fáctico.

Tanto el neoconsecuencialismo como las versiones solventes de la deontología pueden considerarse éticas de la responsabilidad, ya que en ambos casos la valoración de la acción está abierta al futuro, a la interrelación entre actos y consecuencias, entre los principios y su impacto en el mundo. Ni el consecuencialismo ampliado se desentiende de la relatividad del agente, ni la deontología en el marco de una ética de la responsabilidad piensa que los principios estén para proteger la pureza del sujeto. En ambas perspectivas los juicios morales se refieren a un mundo, el que hay, en que los agentes morales tienen compromisos con los demás y con cómo sea ese mundo en general. No obstante, las éticas deontológicas contienen una intuición sustancialmente distinta de la del consecuencialismo ampliado, y es el carácter específico, sustantivo, del mal moral. La deontología defiende que hay acciones buenas por sí mismas, y eso es tanto como decir que también hay acciones malas por sí mismas. No sólo el bien es independiente de las consecuencias, sino que también lo es el mal. Tal como dice Nagel (1988, p. 163), "la esencia del mal es que debería repelernos [...]; eso es lo que "maldad' significa", y ese rechazo tiene que ser, como la sangre en las manos de Caín, indeleble. Y esto es algo que el consecuencialismo, por más que se amplíe, no puede evitar; es la constatación de que el mundo no se adecúa a las exigencias de la razón práctica. ${ }^{20}$ El consecuencialismo

${ }^{20}$ C. Larmore (1987, p. 150) lo expresa diciendo que "tenemos que vivir con el hecho de que hay obligaciones que no podemos atender. Nuestras posibilidades en el mundo son así demasiado limitadas para lo que sabemos que debemos hacer" (la traducción es mía). Concluye este autor que precisamente porque la diferencia entre las obligaciones deontológicas y las consecuencialistas es irreductible, tal diferencia manifiesta en qué consiste ser agente moral. Fue Max Weber quien primero 
y la deontología manifiestan dimensiones profundas y básicas de la razón práctica humana, de la misma forma que también lo hace la propia diferencia entre ambas clases de teoría ética.

\section{BIBLIOGRAFÍA}

Anderson, E.S., 1993, Value in Ethics and Economics, Harvard University Press, Harvard.

Anscombe, G.E.M., 1958, "Modern Moral Philosophy", Philosophy, vol. 33, no. 124, pp. 1-19; también disponible en: <http://www.philosophy.unce. edu/mleldrid/SzCMT/mmp.html> [última consulta: 04/09/2009]. [Versión en castellano: "Filosofía moral moderna", en M. Platts (comp.), Conceptos éticos fundamentales, Instituto de Investigaciones FilosóficasUNAM, México, 2006, pp. 27-53.]

no: Intención, trad. A.I. Stellino, Paidós/Instituto de Investigaciones Filosóficas-UNAM, Barcelona, 1991.]

Aquino, T., 1954, Suma teológica, trad. Francisco Barbado, La Editorial Católica, Madrid (Biblioteca de Autores Cristianos), 4 vols.

Baron, M., P. Pettit y M. Slote (comps.), 1997, Three Methods of Ethics: A Debate, Blackwell, Oxford.

Bentham, J., 2005, The Collected Works of Jeremy Bentham, Oxford University Press, Oxford.

Brandt, R.B., 1959, Ethical Theory, Prentice-Hall, Englewood Cliffs.

Broome, J., 1992, "Deontology and Economics", Economics and Philosophy, vol. 8, no. 2, pp. 269-282.

- 1991, Weighing Goods: Equality, Uncertainity and Time, Blackwell, Oxford.

Carrasco, M., 2002, Consecuencias, agencia y moralidad, Comares, Granada.

Conly, S., 1985, “The Objectivity of Morals and the Subjectivity of Agents", American Philosophical Quarterly, vol. 22, no. 4, pp. 275-286.

Darwall, S. (comp.), 2006, Deontology, Blackwell, Oxford.

- (comp.), 2003, Consequentialism, Blackwell, Oxford.

Foot, P., 1985, "Utilitarianism and the Virtues", Mind, vol. 94, no. 374, pp. 196-209.

Frankena, W.K., 1973, Ethics, Prentice Hall, Englewood Cliffs; también disponible en: <http://www.ditext.com/frankena/ethics.html> [última consulta: 04/09/2009].

formuló esta idea: precisamente porque es genuina la oposición entre la ética de la convicción (la deontología estrecha en terminología seniana), y la ética de la responsabilidad (deontología y consecuencialismo amplios), precisamente porque hay un núcleo de verdad en la ética de la convicción, ésta y la ética de la responsabilidad "no son términos absolutamente opuestos sino elementos complementarios que han de concurrir para formar al hombre auténtico" (Weber 1992, p. 176). 
Griffin, J., 1996, Value Judgment. Improving Our Ethical Beliefs, Oxford University Press, Oxford.

- 1992, "The Human Good and the Ambitions of Consequentialism", Social Philosophy and Policy, vol. 9, no. 2, pp. 118-132.

Hare, R.M., 1993, “Could Kant Have Been a Utilitarian?”, Utilitas, vol. 5, no. 1 , pp. 1-16.

Harsanyi, J., 1982, "Morality and the Theory of Rational Behaviour", en A. Sen y B. Williams (comps.), Utilitarianism and Beyond, Cambridge University Press, Cambridge, pp. 39-62.

Hart, H.L.A., 1967, Punishment and Responsibility. Essays in the Philosophy of Law, Clarendon Press, Oxford.

Jackson, F., 1991, "Decision-Theoretic Consequentialism and the Nearest and Dearest Objection", Ethics, vol. 101, no. 3, pp. 461-482.

Kagan, S., 1998, Normative Ethics, Westview Press, Boulder.

- 1989, The Limits of Morality, Oxford University Press, Oxford.

Kant, I., 1993, Crítica de la razón pura, trad. P. Ribas, Alfaguara, Madrid.

- 1990, Fundamentación de la metafísica de las costumbres, trad. M. García Morente, Porrúa, México.

Lara, F. y P. Francés (comps.), 2004, Ética sin dogmas: racionalidad, consecuencias y bienestar en el utilitarismo contemporáneo, Biblioteca Nueva, Madrid.

Larmore, C., 1987, Patterns of Moral Complexity, Cambridge University Press, Cambridge.

MacIntyre, A., 2001, "Doing Away with Double Effect", Ethics, vol. 111, no. 2, pp. 219-255.

Mackie, J., 1977, Ethics. Inventing Right and Wrong, Penguin, Harmondsworth.

McNaughton, D., y P. Rawling, 1991, “Agent Relativity and the DoingHappening Distinction", Philosophical Studies, vol. 63, no. 2, pp. 167185.

Morgan, S., 2009, "Can There Be a Kantian Consequentialism?", Ratio, vol. 22 , pp. 19-40.

Mulgan, T., 2001, The Demands of Consequentialism, Clarendon Press, Oxford.

Nagel, T., 1988, “Autonomy and Deontology”, en Scheffler 1988, pp. 142173.

- 1980, The Limits of Objectivity, The University of Utah Press, Salt Lake City.

Nino, C.S., 1992, "Consecuencialismo, debate ético y jurídico", Telos: Revista Iberoamericana de Estudios Utilitaristas, vol. 1, no. 1, pp. 7396.

Otsuka, M., 2009, “The Kantian Argument for Consequentialism", Ratio, vol. 22 , no. 1 , pp. 41-58. 
Parfit, D., en prensa, On What Matters, Oxford University Press, Oxford; una versión preliminar se encuentra disponible en: <http://fas-philosophy.rutgers.edu/chang/Papers/OnWhatMattersl.pdf $>$ [última consulta: 04/09/2009].

-, 1984, Reasons and Persons, Clarendon Press, Oxford.

, 1979, "Prudencia, moralidad y el dilema del prisionero", Diálogo Filosófico, vol. 5, no. 13, pp. 4-30.

Pettit, P. (comp.), 1993, Consequentialism, Dartmouth, Aldershot.

Prichard, H.A., 1912, “Does Moral Philosophy Rest on a Mistake?", Mind, vol. 21, no. 81, pp. 21-37. [Versión en castellano: “¿Descansa la filosofía moral en un error?", en E. Rabossi y F. Salmerón, Ética y análisis, Instituto de Investigaciones Filosóficas-UNAM, México, 1985.]

Putnam, H., 2004, El desplome de la dicotomía hecho valor y otros ensayos, trad. N.B. Goethe, Paidós, Barcelona (Paidós Básica, 121).

Quinn, W., 1989, “Actions, Intentions, and Consequences: the Doctrine of Double-Effect", Philosophy and Public Affairs, vol. 18, no. 4, pp. 334351.

Railton, P., 1984, "Alienation, Consequentialism and the Demands of Morality", Philosophy and Public Affairs, vol. 13, no. 84, pp. 134-171.

Rawls, J., 1971, A Theory of Justice, Oxford University Press, Oxford. [Versión en castellano: Teoría de la justicia, trad. María Dolores González, Fondo de Cultura Económica, México, 1995.]

Ross, W.D., 1930, The Right and the Good, Clarendon Press, Oxford.

Salcedo Megales, G., 1991, "Consecuencialismo e imparcialidad", Revista de Filosofía, vol. 4, no. 5, pp. 163-190.

Scanlon, T.M., 2008, Moral Dimensions: Permissibility, Meaning, Blame, Basic Books, Cambridge.

—_, 2001, "Symposium on Amartya Sen's Philosophy: 3. Sen and Consequentialism", Economics and Philosophy, vol. 17, no. 1, pp. 39-50.

Scheffler, S. (comp.), 1988, Consequentialism and its Critics, Oxford University Press, Nueva York.

- 1982 , The Rejection of Consequentialism, Oxford University Press, Oxford.

Sen, A., 2000, "Consequential Evaluation and Practical Reason", The Journal of Philosophy, vol. 97, no. 9, pp. 477-502.

- , 1993, "Positional Objectivity", Philosophy and Public Affairs, vol. 22, no. 2, pp. 126-145.

— , 1983, "Evaluator Relativity and Consequential Evaluation", Philosophy and Public Affairs, vol. 12, no. 2, pp. 113-132.

- , 1982, "Rights and Agency", Philosophy and Public Affairs, vol. 11, no. 1, pp. 3-39.

__ 1979a, "Informational Analysis of Moral Principles", en R. Harrison (comp.), Rational Action, Cambridge University Press, Cambridge, pp. 115-132. 
Sen, A., 1979b, "Utilitarianism and Welfarism", The Journal of Philosophy, vol. 76, no. 9, pp. 463-489.

-, 1977, "On Weights and Measures: Informational Constraints in Social Welfare Analysis", Econometrica: Journal of the Econometric Society, vol. 45, no. 7, pp. 1539-1572.

_- 1974, "Informational Bases of Alternatives Welfare Approaches: Aggregation and Income Distribution", Journal of Public Economics, vol. 3, no. 4, pp. 387-403.

_- 1967, "The Nature and Classes of Prescriptive Judgments", Philosophical Quarterly, vol. 17, no. 66, pp. 46-62.

Unamuno, M., 1997, San Manuel Bueno, mártir, ed. Mario J. Valdés, Cátedra, Madrid (Letras Hispánicas, 95).

Walsh, V., 1996, Rationality, Allocation, and Reproduction, Clarendon Press, Oxford.

Weber, M., 2002, Economía y sociedad: esbozo de sociología comprensiva, trad. J. Medina, Fondo de Cultura Económica, México.

— Libro de Bolsillo, 71).

Williams, B., 1985, Ethics and the Limits of Philosophy, Fontana, Londres. [Versión en castellano: La ética y los límites de la filosofía, trad. Luis Castro Leiva, Monte Ávila, Caracas, 1997.]

—_, 1973, "A Critique of Utilitarianism", en J.J.C. Smart y B. Williams (comps.), Utilitarianism: For and Against, Cambridge University Press, Cambridge, pp. 77-150.

Zimmerman, D., 1981, "Hierarchical Motivation and Freedom of the Will", Pacific Philosophical Quarterly, vol. 62, no. 4, pp. 354-368.

Recibido el 4 de septiembre de 2009; aceptado el 24 de marzo de 2010. 